\title{
Hearing dysfunction in patients with Neuro-Sjögren: a cross- sectional study
}

\author{
Tabea Seeliger ${ }^{1}$, Lena Bönig ${ }^{1}$, Torsten Witte ${ }^{2}$, Thea Thiele ${ }^{2}$, Anke Lesinski-Schiedat ${ }^{3}$, Martin Stangel ${ }^{1}$, \\ Thomas Lenarz ${ }^{3}$, Nils Christian Prenzler ${ }^{3 \#}$, Thomas Skripuletz ${ }^{1 \#}$
}

${ }^{1}$ Department of Neurology, Hannover Medical School, Hannover, Germany; ${ }^{2}$ Department of Clinical Immunology \& Rheumatology, Hannover Medical School, Hannover, Germany; ${ }^{3}$ Department of Otolaryngology, Hannover Medical School, Hannover, Germany

Contributions: (I) Conception and design: M Stangel, T Lenarz, T Skripuletz; (II) Administrative support: T Witte, A Lesinski-Schiedat, T Lenarz; (III) Provision of study materials or patients: T Seeliger, L Bönig, T Lenarz, NC Prenzler; (IV) Collection and assembly of data: T Seeliger, L Bönig, T Thiele, A Lesinski-Schiedat, NC Prenzler; (V) Data analysis and interpretation: T Seeliger, L Bönig, A Lesinski-Schiedat; (VI) Manuscript writing: All authors; (VII) Final approval of manuscript: All authors.

\#These authors contributed equally to this work.

Correspondence to: Prof. Dr. med. Thomas Skripuletz. Department of Neurology, Hannover Medical School, Carl-Neuberg-Str. 1, 30625 Hannover, Germany. Email: Skripuletz.Thomas@MH-Hannover.de.

\begin{abstract}
Background: Sjögren's syndrome is an immunologically mediated disease with salivary and lacrimal gland destruction characterised by typical sicca symptoms of dry mouth and eyes. Awareness of extraglandular neurological manifestations such as polyneuropathy and affection of cranial nerves is rising. Hearing loss as consequence of involvement of the vestibulocochlear nerve presents a severe disability. The exact prevalence and nature of hearing dysfunction in patients with Neuro-Sjögren has been insufficiently evaluated to date.

Methods: Thirty patients with Sjögren's syndrome (ACR-EULAR classification criteria) and polyneuropathy were included in the study in the time period between 11/2016 and 03/2018. The median age was 59 years and $57 \%$ were females. Auditory function was investigated by pure tone audiometry, Freiburg speech comprehension audiometry, transient evoked otoacoustic emissions and brainstem evoked response audiometry.

Results: Pure tone audiometry revealed hearing loss in 10/30 patients (33\%) with severity ranging from mild in most patients (60\%) to severe in $10 \%$. In addition, pathological audiometric test findings showed retrocochlear auditory dysfunction in 14 further patients. In total, $24 / 30$ patients (80\%) showed pathological test results on audiometric testing suggesting hearing dysfunction.

Conclusions: In conclusion, our results show that hearing dysfunction as a possible consequence of cranial neuropathy in patients with Neuro-Sjögren has been underestimated in previous studies.
\end{abstract}

Keywords: Sjögren's syndrome; Neuro-Sjögren; cochlear nerve; cranial nerve; polyneuropathy; neuropathy

Submitted Feb 23, 2020. Accepted for publication Jul 09, 2020.

doi: 10.21037/atm-20-1856

View this article at: http://dx.doi.org/10.21037/atm-20-1856

\section{Introduction}

Sjögren's syndrome is an autoimmune inflammatory disease affecting primarily lacrimal and salivary glands, which causes the pathognomonic sicca syndrome with dry eyes and dry mouth (1). However, extraglandular manifestations and especially neurologic symptoms are increasingly reported
(2-5). Among those, involvement of the peripheral nervous system is the most prevalent [up to $62 \%$ of symptomatic patients (6)], while cranial nerve affection has been described for up to $41 \%$ in neurologic cohorts. Impairment of the VIIIth cranial nerve causing hearing loss is mostly reported in single cases only (7-9) and was only more frequent in one cohort of patients with Sjögren's syndrome 
and neuropathy (18\%) (10) and one other cohort of rheumatologic patients with Sjögren's syndrome (46\%) (11). In order to facilitate recognition, awareness and therapeutic approaches, we systematically assessed the prevalence and characterization of auditory deficiencies in patients with Sjögren's syndrome and polyneuropathy.

We present the following article in accordance with the STROBE reporting checklist (available at http://dx.doi. org/10.21037/atm-20-1856).

\section{Methods}

\section{Patient selection}

Auditory investigations were performed in patients with Sjögren's syndrome and polyneuropathy treated as inpatients at the Department of Neurology of the Hannover Medical School between 11/2016 and 03/2018 (cross sectional study design). Sjögren's syndrome diagnosis was reconfirmed for every patient via validation of the current ACR/EULAR classification criteria (12).

\section{Diagnostic procedures}

Auditory function was evaluated by 5 subtests which were performed for each ear separately.

Pure tone audiometry was performed by presentation of a pure (one frequency) tone stimulus to the patient in rising volume. Patients were instructed to indicate once the stimulus was perceived, to determine the lowest audible volume threshold. Initial testing was performed for $1,000 \mathrm{kHz}$ (a frequency that is easily perceived); then other frequencies of $0.25,0.5,0.75,1.5,2.0,3.0,4.0,6.0$ and $8.0 \mathrm{kHz}$ were added in random order. This procedure was applied for air and bone conduction via specific headphones. Average air to bone conduction gap of $\geq 10 \mathrm{~dB}$ was described as sound conduction impairment. Hearing loss was then quantified by calculation of the average air conduction threshold in $\mathrm{dB}$ at $0.5,1,2$ and $4 \mathrm{kHz}$ [pure tone average 4 (PTA4)] (13). The degree of hearing loss was classified according to the recommendations of the European Working Group on Genetics of Hearing Impairment as mild (>20-40 dB), moderate ( $>40-70 \mathrm{~dB})$, severe $(>70$ $95 \mathrm{~dB}$ ) or profound $(>95 \mathrm{~dB})(14)$. A difference in PTA4 of $\geq 15 \mathrm{~dB}$ between both ears was considered an asymmetrical auditory function. The average auditory function of low frequencies was then deviated based on the bone conduction threshold at $0.5 \mathrm{kHz}$. If neighbouring threshold values at
0.25 and/or $1 \mathrm{kHz}$ were lower in comparison to $0.5 \mathrm{kHz}$, they were additionally included into calculation and the average value was taken as representative for the auditory function of low frequencies.

Freiburg audiometry aimed at testing speech comprehension as hearing threshold tends to be optimized once higher associative cognitive functions are involved. Testing was obtained with monosyllable words and twosyllable numbers as stimuli, all of which were presented at 60,80 and $100 \mathrm{~dB}$. Correct comprehension was then protocolled in percent $(0-100 \%)$. A difference in monosyllable word comprehension $60 \mathrm{~dB}$ of $\geq 15 \%$ of the value for the worse ear was considered asymmetrical speech comprehension. Additionally, the volume level for $50 \%$ comprehension of two-syllable numbers was documented as A1 value (in $\mathrm{dB}$ ). Loss of discrimination was evident if comprehension diminished with rising volume.

Transient evoked otoacoustic emissions (TEOAE) represent the functionality of the outer hair cells. Application of an acoustic pulse leads to reflection of sounds in the healthy ear (15). In our cohort, cochlear functionality was assumed, if TEOAE were reproducible in $\geq 50 \%$.

Auditory brainstem response (ABR) potentials can be measured by vertex- and mastoid-placed electrodes following auditory stimuli. Retrocochlear hearing impairment manifests as delayed or missing response and may be caused by acoustic nerve tumour or other acoustic nerve damage. Morphology of the ABR potentials was considered pathological if the interpeak latency between Jewett potentials I and V measured $>0.3$ or if Jewett potential I was missing (16). Additionally, the ABR derived hearing threshold was analysed for relevant discrepancy to the audiometry derived hearing threshold of $>30 \mathrm{~dB}$ (16). ABRs were successfully tested in 21 patients, while in one additional patient, measurements were non evaluable due to technical issues. In the other 8 patients, testing was not possible due to severe gait disability with wheelchair dependency and consecutively inaccessible test room infrastructure.

Tympanometry was additionally performed to exclude middle ear abnormalities, i.e., luxation of the auditory ossicles, serous otitis media or a defect of the ear drum.

\section{Retrococblear auditory dysfunction}

We analysed patients' performance for evidence of retrocochlear auditory dysfunction as parameter for cranial 
Table 1 ACR/EULAR classification criteria for Sjögren's syndrome. Diagnosis of Sjögren's syndrome is recommended with $\geq 4$ points (pts) in total

\begin{tabular}{lc}
\hline ACR/EULAR classification criteria & N [\%] \\
\hline Xerophthalmia (1 pt) & $26 / 30[87]$ \\
Xerostomia (1 pt) & $16 / 30[53]$ \\
SSA (Ro) antibody positive (3 pts) & $13 / 30[43]$ \\
Sialadenitis on minor salivary gland biopsy (3 pts) & $24 / 30[80]$ \\
\hline
\end{tabular}

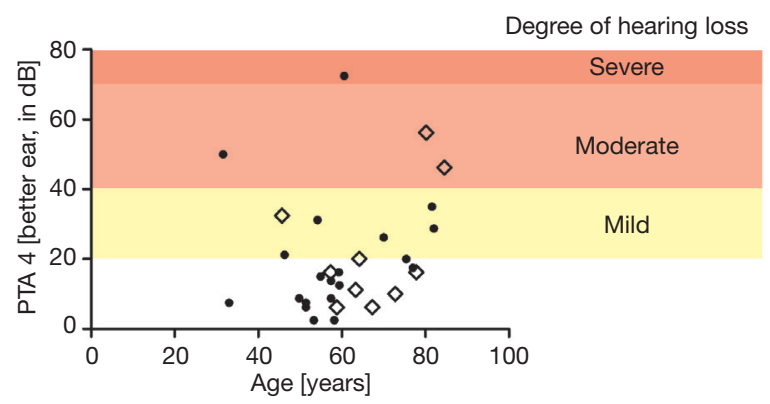

Figure 1 Correlation of age and pure tone average 4 (PTA4) in the better ear with classification of the degree of hearing loss in accordance with the European Working Group on Genetics of Hearing Impairment. Rectangles indicate patients with evidence of retrocochlear auditory dysfunction.

neuropathy, which was diagnosed if one of the following criteria was fulfilled:

(I) Loss of discrimination in the Freiburg speech comprehension test: did the level of comprehension diminish with rising volume?

(II) Discrepancy between comprehension of twosyllable numbers (A1 value) and average auditory function of lower frequencies: was the $\mathrm{A} 1 \geq 20 \mathrm{~dB}$ higher than the calculated average auditory function of lower frequencies?

(III) Pathological ABR morphology (as explained above).

(IV) Discrepancy between the ABR derived and the audiometry derived hearing threshold of $>30 \mathrm{~dB}$.

\section{Ethical approval and informed consent}

The study was approved by the institutional Ethics Committee of the Hannover Medical School (file no. 7460) and was conducted according to the Helsinki Declaration (as revised in 2013). Patients gave their informed consent before participation.

\section{Statistical analysis}

Data were descriptively analysed. Two-sided $t$-tests for heteroskedastic data were used for between group differences, if statistical requirements were met. $\mathrm{P}$ values of $<0.05$ were considered significant. Missing data were descriptively handled.

\section{Results}

\section{Patient characteristics}

In total, 30 patients ( $57 \%$ females, median age 59 years) were included in the study. All patients showed signs of peripheral polyneuropathy and fulfilled the current ACR/ EULAR classification criteria for Sjögren's syndrome (17), which are itemized in Table 1.

\section{Audiometric testing}

All 30 patients received audiometric testing. Pure tone audiometry showed a median PTA4 of $16.3 \mathrm{~dB}$ [interquartile range (IQR), 8.75-28.13] in the better ear, while 10/30 patients $(33 \%)$ presented with hearing loss [threshold $>20 \mathrm{~dB}$ as defined by the European Working Group on Genetics of Hearing Impairment (14)] in the better ear. Among these patients, the degree of hearing loss was mild in $6 / 10$ cases $(60 \%)$, moderate in $3 / 10$ cases $(30 \%)$ and severe in $1 / 10$ cases $(10 \%)$. Correlation of age and audiometry performance is shown in Figure 1. Table 2 shows the comparison of patients with and without hearing loss as detected by pure tone audiometry.

Patients with hearing loss $>20 \mathrm{~dB}$ on pure tone audiometry $(\mathrm{N}=10)$ presented with a mean PTA4 in the better ear of $40 \mathrm{~dB}$ and showed a discrimination loss in $2 / 10$ cases $(20 \%)$ and pathological ABR morphology in $4 / 5$ cases $(80 \%)$, while discrimination loss and pathological ABR morphology overlapped in one patient. A relevant discrepancy of $>30 \mathrm{~dB}$ between the ABR derived and the audiometry derived hearing threshold was found in 1 of those 10 patients (10\%). Evidence of retrocochlear auditory dysfunction was consecutively found in $5 / 10$ patients $(50 \%)$ with hearing loss on pure tone audiometry. Auditory function was asymmetrical in $\geq 1$ audiometric test in $7 / 10$ patients (70\%). 
Table 2 In between group differences: patients with and without hearing loss as detected by pure tone audiometry

\begin{tabular}{lccc}
\hline Variables & Hearing loss on pure tone audiometry & No hearing loss on pure tone audiometry & $P$ value \\
\hline Patients, $\mathrm{n}[\%]$ & $10 / 30[33]$ & $20 / 30[67]$ & $11 / 20[55]$ \\
Females, $\mathrm{n}[\%]$ & $6 / 10[60]$ & 59 & - \\
Median age, years & 65 & 0.57 \\
\hline
\end{tabular}

Table 3 Audiometric testing results for patients with hearing loss and additional patients with retrocochlear auditory dysfunction but without hearing loss on pure tone audiometry

\begin{tabular}{|c|c|c|}
\hline Variables & $\begin{array}{l}\text { Patients with hearing loss on } \\
\text { pure tone audiometry }\end{array}$ & $\begin{array}{l}\text { Additional patients with evidence of } \\
\text { retrocochlear auditory dysfunction (no hearing } \\
\text { loss on pure tone audiometry) }\end{array}$ \\
\hline PTA4 better ear, mean in dB & 40 & 13 \\
\hline Discrimination loss (mono- or bilaterally), n [\%] & 2/10 [20] & $0 / 14[0]$ \\
\hline $\begin{array}{l}\text { Discrepancy of ABR and audiometry derived threshold } \\
>30 \mathrm{~dB}, \mathrm{n}[\%]\end{array}$ & $1 / 5[20]$ & $10 / 14[71]$ \\
\hline TEOAEs abnormal (mono- or bilaterally), n [\%] & $6 / 10[60]$ & $1 / 14[7]$ \\
\hline Asymmetric results in $\geq 1$ audiometric test, $n$ [\%] & 8/10 [80] & $7 / 14[50]$ \\
\hline Left & 34 & 12 \\
\hline \multicolumn{3}{|l|}{ Speech comprehension at $60 \mathrm{~dB}$, mean in $\mathrm{dB}$} \\
\hline Right & 40 & 81 \\
\hline Left & 34 & 86 \\
\hline
\end{tabular}

PTA4, pure tone audiometry 4; ABR, auditory brainstem response; TEOAEs, transient evoked otoacoustic emissions.

Among the other 20 patients without hearing loss on pure tone audiometry, 14 patients (70\%) presented with pathological audiometric test result suggesting retrocochlear auditory dysfunction. In this subgroup, the mean PTA4 of the better ear was $12 \mathrm{~dB}$, discrimination loss was not found, $\mathrm{ABR}$ potentials were pathological in $11 / 14$ cases $(79 \%)$ and a relevant threshold discrepancy was found in $10 / 14$ patients (71\%). Audiometric testing revealed asymmetrical auditory function in $7 / 14$ patients (50\%). Detailed values for patients with hearing loss and for those without hearing loss on pure tone audiometry but with evidence of retrocochlear auditory dysfunction are shown in Table 3.

In total, $24 / 30$ patients. (80\%) showed pathological test results on audiometric testing suggesting hearing dysfunction. In 14 of those 24 affected patients (58\%), hearing loss was not detected by the established threshold testing (PTA4).

\section{Subgroup: patients with retrocochlear auditory dysfunction}

Considering the full cohort, evidence of retrocochlear auditory dysfunction was found in a total of $19 / 30$ patients $(63 \%)$ leading to hearing loss on pure tone audiometry in 5 cases. The pathological findings that were interpreted as evidence of retrocochlear dysfunction are displayed in detail in Figure 2. 


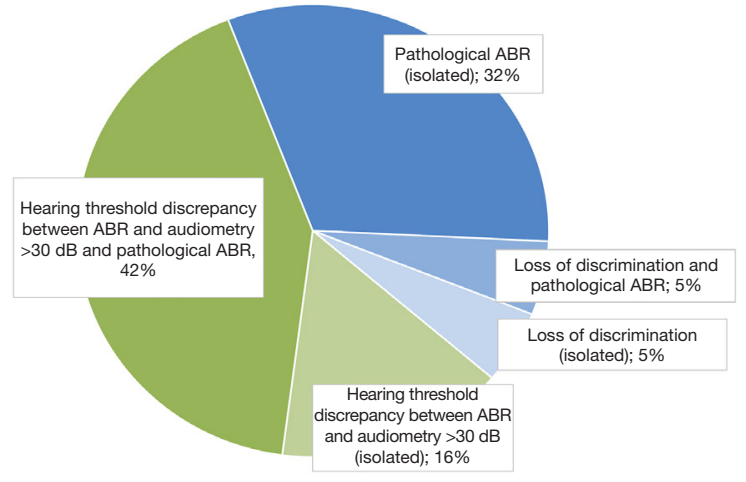

Figure 2 Retrocochlear auditory dysfunction in 19/30 patients (63\%) specified for essential pathological findings. ABR, auditory brainstem response.

\section{Discussion}

Analysis showed that definite hearing loss was evident in $33 \%$ of patients with Sjögren's syndrome and polyneuropathy, while pathological test results on audiometric testing were found in further patients resulting in $80 \%$ of patients in total indicating hearing dysfunction. The severity of hearing loss as detected on pure tone audiometry ranged from mild in most patients $(60 \%)$ to severe in $10 \%$ of patients in our cohort.

Cranial nerve involvement often occurs in patients with Sjögren's syndrome. However, previous studies evaluating cranial nerve impairment predominantly described impaired function of the oculomotor (III), trigeminal (V), facial (VII), glossopharyngeal (IX) and vagus nerve (X) $(8,9,18)$. The involvement of the vestibulocochlear nerve in Sjögren's syndrome-if at all considered-varies in previous studies with rates reported between $2 \%$ and $18 \%(7,9,19)$. Only one Italian study from 1997 claimed hearing loss in a female cohort of 30 rheumatologic patients of $46 \%$ (11). The underestimation of auditory neuropathy in neurologic patients with Sjögren's syndrome might be due to the fact, that on the one hand the extent of subjective auditory dysfunction is difficult to classify [especially in elderly patients (20)] and objective testing on the other hand is complex and therefore often missing in retrospective analyses.

Furthermore, the discrimination between cochlear and retrocochlear hearing loss is challenging and the terminology is under constant revision (21-23). Even with thorough investigation the evidence for retrocochlear dysfunction is always indirect. Anatomical in vivo differentiation of perisynaptic audiopathy through dysfunction of inner hair cells or neuronal damage of the VIIIth cranial nerve cannot be proven via audiometric testing. Only the combination of subjective and objective audiologic tests allows conclusions regarding the topographic origin of hearing loss.

Interestingly, hearing dysfunction did not result in definite hearing loss as detected by the established threshold test (PTA4) in 14/24 patients (58\%) of our cohort. Screening for auditory dysfunction by questionnaire or isolated hearing threshold tests will therefore inevitably lead to underestimation.

The distribution of auditory function was asymmetrical in $80 \%$ of patients with hearing loss and $50 \%$ of patients with retrocochlear auditory dysfunction without hearing loss on pure tone audiometry. Asymmetrical auditory function in general is not a specific finding for inflammatory or other causes, although single sided noise exposure has been described to be associated and schwannomas or causative intracerebral lesions were most frequent if magnetic resonance imaging (MRI) showed underlying pathologies $(24,25)$. Nevertheless, the high rate of asymmetrical auditory function might be due to the fact, that Sjögren's syndrome associated auditory dysfunction might occur in a single sided manner.

Interestingly, 15/19 patients with evidence of retrocochlear auditory dysfunction showed pathological ABRs. ABR potentials are therefore essential in identification of retrocochlear hearing impairment.

The median age of 59 years in patients with Sjögren's syndrome might raise the suspicion of age-related deterioration of auditory function. However, there was no significant age difference between patients with and without hearing loss on pure tone audiometry. In a large prevalence study in Sweden, hearing loss (defined by PTA $4 \geq 25 \mathrm{~dB}$ in the better ear) was reported in only $11.3 \%$ of patients aged 50-60 years (26). We therefore conclude that age related deterioration of auditory function was no significant confounder in our study.

\section{Conclusions}

In conclusion, our data show that auditory dysfunction often occurs in patients with Neuro-Sjögren and that pathologic findings are asymmetrical in $80 \%$ of patients with hearing loss. We thus suggest detailed auditory investigations in patients with Sjögren's syndrome in order to detect auditory dysfunction. 


\section{Acknowledgments}

The authors thank K. Gonsior and the audiometry team of the Hannover Medical School for excellent technical assistance.

Funding: None.

\section{Footnote}

Reporting Checklist: The authors have completed the STROBE reporting checklist. Available at http://dx.doi. org/10.21037/atm-20-1856

Data Sharing Statement: Available at http://dx.doi. org/10.21037/atm-20-1856

Conflicts of Interest: All authors have completed the ICMJE uniform disclosure form (available at http://dx.doi. org/10.21037/atm-20-1856). Dr. MS reports personal fees from Bayer Healthcare, personal fees from Takeda, personal fees from CSL Behring, grants and personal fees from Sanofi-Genzyme, personal fees from Grifols, grants and personal fees from Merck-Serono, personal fees from Roche, grants and personal fees from Novartis, personal fees from Teva, personal fees from MedDay, personal fees from Alexion, grants and personal fees from Biogen, personal fees from Janssen, outside the submitted work. Dr. ThS reports personal fees from Alexion, Bayer Vital, Biogen, Celgene, CSL Behring, Merck, Novartis, Roche, Sanofi Aventis, outside the submitted work. Dr. ThS serves as an unpaid editorial board member of Annals of Translational Medicine from Jan 2020 to Dec 2021. The other authors have no conflicts of interest to declare.

Ethical Statement: The authors are accountable for all aspects of the work in ensuring that questions related to the accuracy or integrity of any part of the work are appropriately investigated and resolved. The study was approved by the institutional Ethics Committee of the Hannover Medical School (file no. 7460) and was conducted according to the Helsinki Declaration (as revised in 2013). Patients gave their informed consent before participation.

Open Access Statement: This is an Open Access article distributed in accordance with the Creative Commons Attribution-NonCommercial-NoDerivs 4.0 International License (CC BY-NC-ND 4.0), which permits the noncommercial replication and distribution of the article with the strict proviso that no changes or edits are made and the original work is properly cited (including links to both the formal publication through the relevant DOI and the license). See: https://creativecommons.org/licenses/by-nc-nd/4.0/.

\section{References}

1 Sjögren H. Zur Kenntnis der Keratoconjunctivitis Sicca. Acta Ophthalmol 1933;13:1-39.

2 Moreira I, Teixeira F, Martins Silva A, et al. Frequent involvement of central nervous system in primary Sjögren syndrome. Rheumatol Int 2015;35:289-94.

3 Pars K, Pul R, Schwenkenbecher P, et al. Cerebrospinal Fluid Findings in Neurological Diseases Associated with Sjögren's Syndrome. Eur Neurol 2017;77:91-102.

4 Iwai K, Amo K, Kuki I, et al. An unusual manifestation of Sjögren syndrome encephalitis. Brain Dev 2019;41:217-20.

5 Yuan J, Gong L, Wu H, et al. Case report of primary Sjögren Syndrome with simple trigeminal lesion as initial symptom. J Neuroimmunol 2018;324:126-8.

6 Delalande S, de Seze J, Fauchais AL, et al. Neurologic manifestations in primary Sjögren syndrome: a study of 82 patients. Medicine (Baltimore) 2004;83:280-91.

7 Sireesha Y, Kanikannan M, Pyal A, et al. Patterns of peripheral neuropathy in Sjogren's syndrome in a tertiary care hospital from South India. Neurol India 2019;67:94.

8 Teixeira F, Moreira I, Silva AM, et al. Neurological involvement in Primary Sjögren Syndrome. Acta Reumatol Port;38:29-36.

9 Sivadasan A, Muthusamy K, Patel B, et al. Clinical Spectrum, Therapeutic Outcomes, and Prognostic Predictors in Sjogren's Syndrome-associated Neuropathy. Ann Indian Acad Neurol 2017;20:278-83.

10 Seeliger T, Prenzler NK, Gingele S, et al. Neuro-Sjögren: Peripheral Neuropathy with Limb Weakness in Sjögren's Syndrome. Front Immunol 2019;10:1600.

11 Tumiati B, Casoli P, Parmeggiani A. Hearing loss in the Sjögren syndrome. Ann Intern Med 1997;126:450-3.

12 Shiboski CH, Shiboski SC, Seror R, et al. 2016 American College of Rheumatology/European League Against Rheumatism classification criteria for primary Sjögren's syndrome. Ann Rheum Dis 2017;76:9-16.

13 World Health Organization. Programme for the Prevention of Deafness and Hearing Impairment. (1997). Report on the First Informal Consultation on Future Programme Developments for the Prevention of Deafness and Hearing Impairment. Geneva, 23-24 January 1997. 
Available online: https://apps.who.int/iris/bitstream/ handle/10665/63421/WHO_PDH_97.3.pdf?sequence=1\& isAllowed=y (accessed 29 March 2019).

14 Mazzoli M, Van Camp G, Newton V, et al. Recommendations for the description of genetic and audiological data for families with nonsyndromic hereditary hearing impairment. Available online: http:// www.gene.ucl.ac.uk/nomenclature/ (accessed 28 March 2019).

15 Kemp DT. Stimulated acoustic emissions from within the human auditory system. J Acoust Soc Am 1978;64:1386-91.

16 Hoth S, Lenarz T. Elektrische Reaktionsaudiometrie. 1st edition. Springer Verlag, 1994.

17 Shiboski CH, Shiboski SC, Seror R, et al. 2016 American College of Rheumatology/European League Against Rheumatism classification criteria for primary Sjögren's syndrome. Ann Rheum Dis 2017;76:9-16.

18 Pereira PR, Viala K, Maisonobe T, et al. Sjögren Sensory Neuronopathy (Sjögren Ganglionopathy): Long-Term Outcome and Treatment Response in a Series of 13 Cases. Medicine (Baltimore) 2016;95:e3632.

19 Seeliger T, Prenzler NK, Gingele S, et al. Neuro-Sjögren: Peripheral Neuropathy With Limb Weakness in Sjögren's Syndrome. Front Immunol 2019;10:1600.

Cite this article as: Seeliger T, Bönig L, Witte T, Thiele T, Lesinski-Schiedat A, Stangel M, Lenarz T, Prenzler NC, Skripuletz T. Hearing dysfunction in patients with Neuro-Sjögren: a cross-sectional study. Ann Transl Med 2020;8(17):1069. doi: 10.21037/atm-20-1856
20 Wiley TL, Cruickshankst\$ KJ, Nondahlt DM, et al. SelfReported Hearing Handicap and Audiometric Measures in Older Adults. 2000. Available online: https://www. audiology.org/sites/default/files/journal/JAAA_11_02_03. pdf (accessed 3 April 2019).

21 Batsoulis C, Lesinski-Schiedat A. Sprachaudiometrie in der Begutachtung des Hörvermögens. HNO 2017;65:203-10.

22 Moser T, Predoehl F, Starr A. Review of hair cell synapse defects in sensorineural hearing impairment. Otol Neurotol 2013;34:995-1004.

23 Starr A, Picton TW, Sininger Y, et al. Auditory neuropathy. Brain 1996;119:741-53.

24 Segal N, Shkolnik M, Kochba A, et al. Asymmetric Hearing Loss in a Random Population of Patients with Mild to Moderate Sensorineural Hearing Loss. Ann Otol Rhinol Laryngol 2007;116:7-10.

25 Cueva RA. Auditory Brainstem Response versus Magnetic Resonance Imaging for the Evaluation of Asymmetric Sensorineural Hearing Loss. Laryngoscope 2004;114:1686-92.

26 Johansson MSK, Arlinger SD. Prevalence of hearing impairment in a population in Sweden. Int J Audiol 2003;42:18-28. 